\title{
Fast-monotone Kettenbrüche
}

\author{
Von Folke Ryde
}

Wir betrachten einen $(n+1)$-gliedrigen Kettenbruch der Form

$$
a_{1}+\frac{a_{1} \mid}{\mid a_{2}}+\frac{a_{2} \mid}{\mid a_{3}}+\cdots+\frac{a_{n-1} \mid}{\mid a_{n}}+\frac{a_{n} \mid}{\mid a_{n+1}},
$$

wo die Zahlen $a_{1}, a_{2}, a_{3}, \ldots a_{n-1}, a_{n}, a_{n+1}$ ganze, positive Zahlen sind, die folgende Bedingungen erfüllen $a_{1} \geqq a_{2} \geqq a_{3} \geqq \cdots \geqq a_{n-1} \geqq a_{n}$. Hinsichtlich der Zahl $a_{n+1}$ können folgende Fälle unterschieden werden:

1. $a_{n+1}=1$. Der Kettenbruch mag dann ein monotoner, nicht-wachsender Kettenbruch genannt werden. Ich habe diese Kettenbrüche in einigen Schriften ${ }^{1}$ untersucht.

2. $1<a_{n+1} \leqq 2 a_{n}$.

3. $2 a_{n}<a_{n+1}$. Der Kettenbruch mag dann ein fast-monotoner Kettenbruch genannt werden.

Hinsichtlich des zweiten Falles gilt folgender Satz:

Aus der Kettenbruchentwicklung

$$
a_{1}+\frac{a_{1} \mid}{\mid a_{2}}+\frac{a_{2} \mid}{\mid a_{3}}+\cdots+\frac{a_{n-1} \mid}{\mid a_{n}}+\frac{a_{n} \mid}{\mid a_{n+1}},
$$

wo $a_{1}, a_{2}, a_{3}, \ldots a_{n-1}, a_{n}, a_{n+1}$ solche ganze, positive Zahlen sind, dass die folgenden Bedingungen erfüllt sind: $a_{1} \geqq a_{2} \geqq a_{3} \geqq \cdots \geqq a_{n-1} \geqq a_{n}$ und $1<a_{n+1} \leqq 2 a_{n}$, kann immer durch fortgesetzte Entwicklung der Zahl $a_{n+1}$ wenigstens eine monotone oder fast-monotone Kettenbruchentwicklung erhalten werden.

1 Der Algorithmus der monotonen, nicht.wachsenden Kettenbrüche. Arkiv för matematik, astronomi och fysik. Bd 31 A. N:o 19 (1944). Diese Schrift wird im Folgenden mit M. K. I bezeichnet.

Über die rekursorische Berechnung der monotonen, nicht-wachsenden Kettenbrüche, Arkiv för matematik, astronomi och tysik. Bd $31 \mathrm{~B}$. N:o 12 (1944). Diese Schrift wird im Folgen. den mit M. K. II bezeichnet.

Tafel und Nomogramm der monotonen, nicht-wachsenden Kettenbrüche. Arkiv för matematik, astronomi och fysik. Bd 34 A. N:o 11 (1947). Diese Schrift wird im Folgenden mit M. K. III bezeichnet.

Eine Produktdarstellung der monotonen, nicht-wachsenden Kettenbrüche. Arkiv för matematik, astronomi och fysik. Bd 34 A. N:o 16 (1947). Diese Schrift wird im Folgenden mit M. K. IV bezeichnet. 


\section{F. RXDE, Fast-monotone Kettenbrüche}

Denn, wenn $a_{n+1}$ eine gerade Zahl bedeutet, führt die Entwicklung

$$
a_{n+1}=\frac{1}{2} a_{n+1}+\frac{\frac{1}{2} a_{n+1} \mid}{\mid 1}
$$

zu einer monotonen Entwicklung, da $\frac{1}{2} a_{n+1} \leqq a_{n}$ ist, und, wenn $a_{n+1}$ eine ungerade Zahl $>5$ bedeutet, führt die Entwicklung

$$
a_{n+1}=\left[\frac{1}{2} a_{n+1}\right]+1+\frac{\left[\frac{1}{2} a_{n+1}\right]+1 \mid}{\mid 1}+\frac{1}{\mid\left[\frac{1}{2} a_{n+1}\right]}
$$

zu einer fast-monotonen Entwicklung, weil dann

$$
\left[\frac{1}{2} a_{n+1}\right]+1=\frac{1}{2} a_{n+1}-\frac{1}{2}+1=\frac{1}{2} a_{n+1}+\frac{1}{2} \leqq a_{n} .
$$

Denn zufolge der Bedingung $a_{n+1} \leqq 2 a_{n}$ und weil $a_{n+1}$ eine ungerade Zahl bedeutet, ist $a_{n+1} \leqq 2 a_{n}-1$, das heisst $\frac{1}{2} a_{n+1}+\frac{1}{2} \leqq a_{n}$. Weiterhin ist $\left[\frac{1}{2} a_{n+1}\right]>2$, da $a_{n+1}>5$ ist.

Es erübrigt noch die Fälle $a_{n+1}=5$ und $3 \mathrm{zu}$ behandeln. Im ersten Fall führt die Entwicklung $5=3+\frac{3 \mid}{\mid 1}+\frac{1 \mid}{\mid 1}+\frac{1 \mid}{\mid 1}$ zu einer monotonen Entwicklung, weil $5 \leqq 2 a_{n}-1$ ist, d.h. $3 \leqq a_{n}$. Im zweiten Fall führt die Entwicklung $3=2+\frac{2 \mid}{\mid 1}+\frac{1 \mid}{\mid 1}$ zu einer monotonen Entwicklung, weil $3 \leqq 2 a_{n}-1$ ist, d. $\mathrm{h}$. $2 \leqq a_{n}$.

Da die im vorstehenden Satz erwähnten Kettenbruchentwicklungen immer auf die angegebene Weise zu monotonen, bzw. fast-monotonen Entwicklungen fortgesetzt werden können, können wir im Folgenden von diesem Fall absehen. Dagegen sind die fast-monotonen Kettenbruchentwicklungen als Entwicklungen neuer Art im Verhältnis zu den monotonen Entwicklungen anzusehen, was aus dem folgenden Satz ersichtlich wird:

Aus der fast-monotonen Kettenbruchentwicklung

$$
a_{1}+\frac{a_{1} \mid}{\mid a_{2}}+\frac{a_{2} \mid}{\mid a_{3}}+\cdots+\frac{a_{n-1} \mid}{\mid a_{n}}+\frac{a_{n} \mid}{\mid a_{n+1}}
$$

kann nimmer durch fortgesetzte Entwicklung der Zahl $a_{n+1}$ weder eine neue fastmonotone Entwicklung noch eine monotone Entwicklung erhalten werden.

Denn es ist diesfalls nicht möglich, die Zahl $a_{n+1}$ folgendermassen zu entwickeln

$$
a_{n+1}=b_{1}+\frac{b_{1} \mid}{\mid b_{2}}+\frac{b_{2} \mid}{\mid b_{3}}+\cdots+\frac{b_{m-1} \mid}{\mid b_{m}}+\frac{b_{m} \mid}{\mid b_{m+1}},
$$

wo $b_{1}, b_{2}, b_{3}, \ldots b_{m-1}, b_{m}$ und $b_{m+1}$ ganze, positive Zahlen der Art sind, dass $b_{1} \geqq b_{2} \geqq b_{3} \geqq \cdots \geqq b_{m-1} \geqq b_{m}$ und wo $b_{1} \leqq a_{n}$-ist. Denn unter den angegebenen Bedingungen ist 


$$
b_{2}+\frac{b_{2} \mid}{\mid b_{3}}+\cdots+\frac{b_{m-1} \mid}{\mid b_{m}}+\frac{b_{m} \mid}{\mid b_{m+1}} \geqq 1
$$

und somit $a_{n+1} \leqq 2 b_{1}$, d. h. $a_{n+1} \leqq 2 a_{n}$, was im Widerspruch mit der Annahme steht, dass der gegebene Kettenbruch ein fast-monotoner ist.

Im Folgenden beabsichtige ich die fast-monotonen Kettenbrüche der Hauptsache nach in gleicher Weise zu untersuchen, wie ich in den obenerwähnten Schriften M. K. I--IV die monotonen Kettenbrüche behandelt habe.

Die Auseinandersetzungen in M. K. I $\$ ₫ 1-6$ behalten - mutat is mutand is - ihre Gültigkeit auch rücksichtlich der fast-monotonen Kettenbrüche. Folgende Umstände müssen dabei beachtet werdea:

1. Der Algorithmus führt zum Gclinknennerabbruch bei dem $n$-ten Gelenk nicht nur für $r_{n}<1$ sondern auch für $r_{n}=1$, wo $r_{n}$ der $n$-te Gelenknenner bedeutet.

2. Der Algorithmus führt zum Abschluss bei dem $n$-ten Gelenk, wenn der $n$-te Gelenknenner eine ganze Zahl bedeutet, die grösser als der doppelte Wert der $n$-ten Gelenkzahl ist, d. h. $r_{n}$ ganz und $r_{n}>2 s_{n}$.

3. Bei jedem Schritt des Algorithmus, wie er in M. K. I $\S 5$ formuliert worden ist, muss die Möglichkeit einer Gelenkzahl $\left[r_{n}-\frac{1}{2}\right]+1$ beachtet werden: Wenn nämlich $1<r_{n}<\left[r_{n}\right]+\frac{1}{2}$ und $\left[r_{n}\right]:\left(r_{n}-\left[r_{n}\right]\right)$ eine ganze Zahl bedeutet, liegt Abschluss bei dem $(n+1)$-ten Gelenk vor für $s_{n+1}=\left[r_{n}\right]$, d. h. $s_{n+1}=$ $=\left[r_{n}-\frac{1}{2}\right]+1$, falls dieser Wert nicht durch die Monotoniebedingung ausgeschlossen ist. Eine noch grössere Gelenkzahl $s_{n+1}$ ist wegen der Beziehung $r_{n}<\left[r_{n}\right]+1$ ausgeschlossen. Wegen derselben Beziehung ist die Möglichkeit einer Gelenkzahl $s_{n+1}>\left[r_{n}-\frac{1}{2}\right]$ im Fall $r_{n} \geqq\left[r_{n}\right]+\frac{1}{2}$ ausgeschlossen. Bei einer Gelenkzahl $s_{n+1} \leqq\left[r_{n}-\frac{1}{2}\right]$ ist Abschluss bei dem $(n+1)$-ten Gelenk ausgeschlossen. Denn diesfalls wird der $(n+1)$-te Gelenknenner $\leqq s_{n+1}$, falls $1<r_{n}<\left[r_{n}\right]+\frac{1}{2}$ ist, woraus nämlich folgt, dass $\left[r_{n}--\frac{1}{2}\right] \leqq\left[r_{n}\right]-1$ ist, und somit $s_{n+1} \leqq\left[r_{n}-\frac{1}{2}\right] \leqq$ $\leqq\left[r_{n}\right]-1 \leqq r_{n}-1$. Ebenso wird der $(n+1)$-te Gelenknenner $\leqq 2 s_{n+1}$, falls $r_{n} \geqq\left[r_{n}\right]+\frac{1}{2}$ ist, da immerfort $\left[r_{n}\right]+1>r_{n}$ ist, woraus folgt, dass $\left[r_{n}-\frac{1}{2}\right]=\left[r_{n}\right]$ und somit $s_{n+1} \leqq\left[r_{n}-\frac{1}{2}\right]=\left[r_{n}\right] \leqq r_{n}-\frac{1}{2}$. (Der $(n+1)$-te Gelenknenner, $r_{n+1}$, ist mit dem $n$-ten Gelenknenner, $r_{n}$, durch die Beziehung

$$
r_{n}=s_{n+1}+\frac{s_{n+1}}{r_{n+1}}
$$

verknüpft.) Vgl. die Erörterungen in M. K. I $\S 2$ und 3 oder den letzten Satz dieser Abteilung, woraus ebenso folgt, dass Abschluss nur bei einer Gelenkzahl $\left[r_{n}-\frac{1}{2}\right]+1$ auftreten kann.

4. Wenn im Fall $1<r_{n}<\left[r_{n}\right]+\frac{1}{2}$ ins besondere $\left[r_{n}\right]=1$, d. h. $1<r_{n}<3 / 2$ ist, liegt Abschluss bei dem $(n+1)$-ten Gelenk vor, wenn und nur wenn $r_{n}$ eine Zahl der Form $1+1 / m$ mit $m$ ganz und $>2$ ist. Wenn dagegen $\left[r_{n}\right]=2$, 


\section{F. RYDE, Fast-monotone Kettenbrïche}

d. h. $2<r_{n}<5 / 2$ ist, liegt Abschluss bei dem $(n+1)$-ten Gelenk vor, wenn und nur wenn $r_{n}$ eine Zahl der Form $2+2 / m$ mit $m$ ganz und $>4$ ist. Die genannten Zahlen gehören zu denjenigen Intervallen, in denen sonst Gelenkzahlenabbruch vorliegt.

Eine erhebliche Unarbeitung ist nur bei M. K. I $\S 8$ erforderlich. Es gilt der Satz: Es gibt keine rationale Zahl zwischen 1 und 3, die sich auf mehr als eine Weise in der Form eines fast-monotonen Kettenbruches entwickeln lässt. Hierbei kann die Zahl 3 durch keine grössere Zahl ersetzt werden.

Der letzte Teil des Satzes ist evident. Denn die Zahl $3+\frac{1}{2 m+1}$ gestattet für jedes $m>2$ zwei fast-monotone Entwicklungen, nämlich

$$
3+\frac{3}{\mid 6 m+3} \text { und } 2+\frac{2 \mid}{\mid 1}+\frac{1 \mid}{\mid 1}+\frac{1 \mid}{\mid m} \text {. }
$$

Zudem ist $\lim _{m \rightarrow \infty}\left(3+\frac{1}{2 m+1}\right)=3$.

Der erste Teil des Satzes kann z. B. folgendermassen ermittelt werden: Wir zerspalten das Intervall $1 \leqq r \leqq 3$ in folgende Teilintervalle (die Bezeichnung ist im Verhältnis zu M. K. I geändert)

$$
\begin{array}{llll}
1 \leqq r \leqq 3 / 2 & \text { (A) } & 3 / 2<r<2 & \text { (B) } \\
2 \leqq r \leqq 5 / 2 & \text { (C) } & 5 / 2<r \leqq 3 & \text { (D). }
\end{array}
$$

Der Fall $r=1$ führt zu unmittelbarem Abbruch. Im A-Intervall liegt im übrigen Abbruch bei dem zweiten Gelenk vor, wenn nicht der entsprechende Gelenknenner der Form $1+\frac{1}{n}$ mit $n$ ganz und $>2$ ist, in welchem Fall Abschluss bei dem ersten Gelenk vorliegt. Der entsprechende Kettenbruch wird $\left(1_{1}: n\right)$ mit $n$ ganz und $>2$ bezeichnet. Der Fall $r=2$ führt zum Abbruch bei dem ersten Gelenk. Im übrigen liegt Abbruch bei dem zweiten Gelenk im C-Intervall vor, wenn nicht der entsprechende Gelenknenner der Form $2+\frac{2}{n}$ mit $n$ ganz und $>4$ ist, in welchem Fall Abschluss bei dem ersten Gelenk vorliegt. Der entsprechende Kettenbruch wird $\left(2_{1}: n\right)$ mit $n$ ganz und $>4$ bezeichnet.

Wenn $r$ zum B-Intervall gehört, kommt der oben im Mom. 3 erwähnte Fall bei dem ersten Schritt nicht vor. $-\left[-\frac{1}{2} r\right]$ wird $=1$ und $\left[r-\frac{1}{2}\right]=1 . \quad s_{1}$ ist nach M. K. I $\S 5$ eindeutig bestimmt und gleich 1 . Für $r_{1}$ ergibt sich dann, da $r=s_{1}+s_{1} / r_{1}$ ist, $1<r_{1}<2$. B-Intervall, bzw. A-Intervall mit nachfolgendem Abbruch oder Abschluss liegt vor. In der Fortsetzung kommen auch nur dieselben Intervalle vor. Die einzigen Zahlen im B-Intervall, die sich in der Form eines fast-monotonen Kettenbruches entwickeln lassen, sind die Zahlen $\left(1_{l}: n\right)$ mit $l$ ganz und $>1$ und $n$ ganz und $>2$.

Wenn $r$ zum D-Intervall gehört, kommt ebenso der oben im Mom. 3 erwähnte Fall bei dem ersten Schritt nicht vor. $-\left[-\frac{1}{2} r\right]$ wird $=2$ und $\left[r-\frac{1}{2}\right]=2 . \quad s_{1}$ ist eindeutig bestimmt und gleich 2. Für $r_{1}$ ergibt sich $2 \leqq r_{1}<4$. C-Intervall, D-Intervall, der Fall $3<r_{1}<7 / 2$ oder der Fall $7 / 2 \leqq r_{1}<4$ liegt vor. Im Fall $3<r_{1}<7 / 2$, der vorliegt, wenn $18 / 7<r<8 / 3$, 
liegt der in M. K. I $\S 4$ behandelte Fall mit $u=1$ vor. $-\left[-\frac{1}{2} r_{1}\right]$ wird $=2$ und $\left[r_{1}-\frac{1}{2}\right]-([r]-u-1)$ ergibt sich gleich 2. Somit wird auch $s_{2}$ eindeutig bestimmt und gleich 2. Wegen der Monotoniebedingung ist hier der oben im Mom. 3 erwähnte Fall ausgeschlossen. Für $r_{2}$ ergibt sich $4 / 3<r_{2}<2$, d. h. entweder ein Teilintervall des A-Intervalls oder das B-Intervall. Doch gibt es im Teilintervall $4 / 3<r \leqq 3 / 2$ des A-Intervalls keinen Abschluss-Fall. Die entsprechenden fast-monotonen Kettenbrüche sind der Form $\left(2_{2} l_{l}: n\right)$ mit $l$ ganz und $>1$ und $n$ ganz und $>2$. - Im Fall $7 / 2 \leqq r_{1}<4$, der vorliegt, wenn $5 / 2<r \leqq 18 / 7$, liegt der in M. K. I $\S 4$ behandelte Fall mit $u=0$ vor. $--\left[-\frac{1}{2} r_{1}\right]$ wird $=2$ und $\left[r_{1}-\frac{1}{2}\right]-([r]-u-1)$ ergibt sich gleich 2. Somit wird auch $s_{2}$ eindeutig bestimmt und gleich 2. Für $r_{2}$ ergibt sich $1<r_{2} \leqq 4 / 3$, d. h. ein Teilintervall des A-Intervalls. Die entsprechenden fast-monotonen Kettenbrüche sind der Form $\left(2_{2} 1_{1}: n\right)$ mit $n$ ganz und $>2$. - Wenn $r_{1}$ zum CIntervall gehört, sind die entsprechenden fast-monotonen Kettenbrüche der Form $\left(2_{2}: n\right)$ mit $n$ ganz und $>4$. - Wenn zuletzt $r_{1}$ zum D-Intervall gehört, wiederholt sich der Vorgang. Die entsprechenden fast-monotonen Kettenbrïche sind entweder der Form $\left(2_{m} 1_{l}: n\right)$ mit $m$ ganz und $>2$ und $l$ ganz und $\geqq 1$ und $n$ ganz und $>2$ oder der Form $\left(2_{m}: n\right)$ mit $m$ ganz und $>2$ und $n$ ganz und $>4$.

Wie wir gesehen haben, werden die brauchbaren Gelenkzahlen immer eindeutig bestimmt, wenn $1 \leqq r \leqq 3$. Die entsprechenden fast-monotonen Kettenbrüche sind entweder der Form $\left(1_{l}: n\right)$ mit $l$ ganz und $\geqq 1$ und $n$ ganz und $>2$ oder der Form $\left(2_{m}: n\right)$ mit $m$ ganz und $\geqq 1$ und $n$ ganz und $>4$ oder der Form $\left(2_{m} 1_{l}: n\right)$ mit $m$ ganz und $\geqq 2$ und $l$ ganz und $\geqq 1$ und $n$ ganz und $>2$.

Es gilt folgender Satz: Die notwendige und hinreichende Bedingung für einen fast-monotonen Abschluss bei dem $n$-ten Gelenk ${ }^{1}$ kann folgenderweise mittelst des $(n-1)$-ten Gelenknenners ausgedrückt werden: Wenn der $(n-1)$-te Gelenknenner als ein irreduzibler Bruch $\frac{p}{q}$ geschrieben wird, muss $p$ einen Faktor enthalten, der $\equiv 1(\bmod q)$ ist, während das Produkt der anderen Faktoren $<\frac{1}{2} q$ ist.

Denn, wenn $r_{n-1}=s_{n}+\frac{s_{n}}{N}$ ist, so liegt fast-monotoner Abschluss bei dem $n$-ten Gelenk vor, wenn $N$ eine ganze, positive Zahl $>2 s_{n}$ ist. Es ergibt sich dann $r_{n-1}=\frac{p}{q}=\frac{s_{n}(N+1)}{N}$. Da $\frac{p}{q}$ ein irreduzibler Bruch ist, kann $N=q t$ geschrieben werden, wo $t$ eine ganze, positive Zahl bedeutet. Es ergibt sich dann $\frac{p}{q}=\frac{s_{n}(q t+1)}{q t} \cdot$ Da $q t+1 \neq 0(\bmod t)$ ist, muss $s_{n} \equiv 0(\bmod t)$ sein. Es ergibt sich $p=\frac{s_{n}}{t}(q t+1) . \quad p$ enthält somit einen Faktor, nämlich $q t+1$, der $\equiv 1$ $(\bmod q)$ ist, während das Produkt der anderen Faktoren, d. h. $\frac{s_{n}}{t}$ kleiner als $\frac{1}{2} q$ ist, denn $N=q t$ ist $>2 s_{n}$.

\footnotetext{
${ }^{1}$ Die Erfüllung der Monotoniebedingung wird vorausgesetzt.
} 
Wenn anderseits $r_{n-1}=-\frac{p}{q}$, wo $p$ und $q$ teilerfremd sind, wo $p$ einen Faktor, $p_{1}$, enthält, der $\equiv 1(\bmod q)$ ist, während das Produkt der anderen Faktoren, $p_{2}$, kleiner als $\frac{1}{2} q$ ist, so ergibt sich

$$
r_{n-1}=\frac{p_{1} p_{2}}{q}=\frac{(q t+1) p_{2}^{\prime}}{q}=\frac{(q t+1) p_{2} t}{q t}=p_{2} t+\frac{p_{2} t}{q t}
$$

wo die ganze, positive Zahl $q t$ folgende Bedingung erfüllt $q t>2 p_{2} t$, da $q>2 p_{2}$ ist. Dann liegt fast-monotoner Abschluss bei dem Gelenk $p_{2} t+p_{2} t \mid$ vor.

Die Gelenkzahlen 1 und 2 als letzte Gelenkzahlen nehmen hierbei keine Sonderstellung ein.

\section{II}

Es gilt folgender Satz, der das Analogon des Satzes in M. K. IV, Abschn. II ist und der sich in gleicher Weise beweisen lässt:

Eine notwendige und hinreichende Bedingung für die Darstellbarkeit der rationalen Zahl $r$ durch einen $(n+1)$-gliedrigen, fast-monotonen Kettenbruch ist, dass $r$ in folgender Weise in Faktoren zerlegt werden kann

$$
r=\varkappa_{0}\left(1+\frac{1}{\varkappa_{1}}\right)\left(1-\frac{1}{\varkappa_{2}}\right)\left(1+\frac{1}{\varkappa_{3}}\right) \cdots\left(1+\frac{(-1)^{n-1}}{\varkappa_{n}}\right),
$$

wo $\varkappa_{0}, \varkappa_{1}, \varkappa_{2}, \varkappa_{3}, \ldots \varkappa_{n}$ rationale Zahlent sind, die folgende Bedingungen erfüllen:

1. $x_{0}, x_{1}, \frac{x_{2}-x_{1}-1}{x_{1}+1}$ und $\frac{\left(x_{i}+1-x_{i}+(-1)^{i}\right)\left(x_{i}-x_{i-1}+\left(-1,{ }^{i-1}\right)\right.}{x_{i}\left(x_{i}+\left(-1 i^{i-1}\right)\right.}$ für $i=2,3, \ldots n-1$ sollen ganze, positive Zahlen sein.

2. Folgende Ungleichungen sollen erfüllt sein:

$$
\begin{aligned}
& x_{1} \leqq x_{0} \\
& x_{2} \leqq x_{1}\left(x_{1}+1\right)+x_{1}+1 \\
& x_{3} \leqq \frac{x_{2}\left(x_{2}-1\right)}{x_{1}+1}+x_{2}-1
\end{aligned}
$$

und allgemein für $i=3,4, \ldots n-2$

$$
x_{i+1} \leqq \frac{x_{i}\left(x_{i}+(-1)^{i-1}\right)}{x_{i-1}\left(x_{i-1}+(-1)^{i-2}\right)}\left(x_{i-1}-x_{i-2}+(-1)^{i-2}\right)+x_{i}+(-1)^{i-1}
$$

und schliesslich für $n>3$

$$
\varkappa_{n}>2 \cdot \frac{x_{n-1}\left(x_{n-1}+(-1)^{n-2}\right)}{x_{n-2}\left(x_{n-2}+(-1)^{n-3}\right)} \cdot\left(x_{n-2}-x_{n-3}+(-1)^{n-3}\right)+x_{n-1}+(-1)^{n-2} .
$$


ARKIV För MATEMATIK. Bd 1 nr 4

Für $n=1,2$, und 3 soll die letzte Ungleichung durch bzw.

und

$$
\begin{aligned}
& x_{1}>2 x_{0} \\
& x_{2}>2 x_{1}\left(x_{1}+1\right)+x_{1}+1
\end{aligned}
$$

$$
\varkappa_{3}>2 \frac{x_{2}\left(\varkappa_{2}-1\right)}{x_{1}+1}+\varkappa_{2}-1
$$

ersetzt werden.

Für $n=1$ wiederfinden wir den letzten Satz in I.

\section{III}

Hinsichtlich der in M. K. II hergeleiteten Rekursionsformeln, womit die Anzahl der monotonen, nicht-wachsenden Kettenbrüche berechnet werden kann, sei Folgendes angeführt: Die Anzahl der fast-monotonen Kettenbruchentwicklungen der rationalen Zahl $r=\frac{q k+l-q}{q}-$ wir bezeichnen sie $N m^{\prime}(r)$ kann mit Hilfe folgender Rekursionsformeln berechnet werden:

Es gilt

$$
N m^{\prime}(1)=0
$$

und für $k$ ganz und $>2$

$$
N m^{\prime}(k-1)=\sum_{s=-\left[-\frac{k-1}{2}\right]}^{s=k-2} N m^{\prime}\left(\frac{s}{k-1-s}\right)
$$

Es gilt weiterhin

$$
N m^{\prime}\left(\frac{q k+l-q}{q}\right)=\sum_{s=-\left[-\frac{q k+l-q}{2 q}\right]}^{s=k-2} N m^{\prime}\left(\frac{s q}{q k+l-q-s q}\right)+\left\{\frac{k-1}{l}\right\}
$$

unter folgenden Bedingungen

1. $q=$ einer ganzen $Z a h l \geqq 3$,

2. $k=$ einer ganzen $Z a h l \geqq 2$,

3. $l=$ einer ganzen, positiven Zahl,

4. $l$ und $q$ sind teilerfremde Zahlen,

5. $\frac{l}{q}<\frac{1}{2}$, 
F. RYDE, Fast-monotone Kettenbrüche

6. $\left\{\begin{array}{c}k-1 \\ l\end{array}\right\}=1$, wenn $k-1 \equiv 0(\bmod l)$, wobei der Wert $l=1$ nicht ausgeschlossen ist, und

$\left\{\begin{array}{c}k-1 \\ l\end{array}\right\}=0$, wenn $k-1 \neq 0(\bmod l)$.

Für $k=2$ und 3 fällt die Summe auf der rechten Seite in Formel $\left(\mathrm{A}_{3}\right)$ fort.

Dagegen gilt

$$
N m^{\prime}\left(\frac{q k+l-q}{q}\right)=\sum_{s=-\left[-\frac{q k+l-q}{2 q}\right]}^{s=k-1} N m^{\prime}\left(\frac{s q}{q k+l-q-s q}\right)
$$

unter folgenden Bedingungen:

1. $q=$ einer ganzen $Z a h l \geqq 2$,

2. $k=$ einer ganzen $Z a h l \geqq 2$,

3. $l=$ einer ganzen, positiven $Z a h l$,

4. $\frac{k-1}{k} \leqq \frac{l}{q}<1$.

Es gilt weiterhin

$$
\begin{aligned}
N m^{\prime}\left(\frac{q k+l-q}{q}\right)= & \sum_{s=-\left[-\frac{q k+l-q}{2 q}\right]}^{s=k-2} N m^{\prime}\left(\frac{s q}{q k+l-q-s q}\right)+ \\
& +\sum_{t=-\left[-\frac{(k-1) q}{2 l}\right]}^{t=k-1} N m^{\prime}\left(\frac{t l}{(k-1) q-t l}\right)
\end{aligned}
$$

unter folgenden Bedingungen

1. $q=$ einer ganzen $Z a h l \geqq 2$,

2. $k=$ einer ganzen $Z a h l \geqq 3$,

3. $l=$ einer ganzen, positiven Zahl,

4. $\frac{1}{2} \leqq \frac{l}{q}<\frac{k-1}{k}$.

Für $k=3$ fällt die erste Summe auf der rechten Seite in Formel (C) fort.

Hinsichtlich des Falles $l=0$, d. h. $\frac{q k+l-q}{q}=k-1$, sei zuerst bemerkt, dass $N m^{\prime}(k-1)$ für $k=2$ gleich 0 sein muss, denn eine fast-monotone Kettenbruchentwicklung kann nicht den Schlussnenner 1 haben, was bei den monotonen Kettenbruchentwicklungen der Fall ist. Daher ergibt sich $N m^{\prime}(1)=0$, 
während $N m(1)=1$ ist. - Für $k>2$ gilt die Formel $\left(\mathrm{A}_{2}\right)$. Denn laut dessen, was in M. K. I $\S 2$ angeführt worden ist, können dann nur die folgenden Zahlen als erste Gelenkzahlen auftreten

$$
-\left[\begin{array}{c}
k-1 \\
2
\end{array}\right], \quad-\left[-\frac{k-1}{2}\right]+1, \quad \cdots-[-(k-1)]-1,
$$

d. h. für $k \equiv 1(\bmod 2)$ und $k \geqq 3$ die Zahlen $\frac{k-1}{2}, \frac{k+1}{2}, \ldots k-2$ und für $k \equiv 0(\bmod 2)$ und $k \geqq 4$ die Zahlen $\frac{k}{2}, \frac{k+2}{2}, \ldots k-2$. Wenn die erste Gelenkzahl gleich $k-\varkappa$ gesetzt wird, so ergibt sich

$$
k-1=k-x+\frac{k-\varkappa}{r_{1}},
$$

wo $r_{1}=\frac{k-\varkappa}{k-1-(k-x)}=\frac{k-\varkappa}{\varkappa-1}$. Für $\varkappa \geqq 2$ ist mithin $r_{1} \leqq k-\varkappa$ und jede fast-monotone Entwicklung der Zahl $r_{1}$ kann ohne Monotonieabbruch verwertet werden, denn die erste Gelenkzahl jeder solcher Entwicklung ist dann gewiss $<k-\varkappa$. Die in I Mom. 3 besprochene Möglichkeit einer Gelenkzahl $\left[r-\frac{1}{2}\right]+1$ im Zusammenhang mit fast-monotonem Abschluss ist hier ausgeschlossen.

Hinsichtlich des Falles $l>0$ sei zuerst bemerkt, dass es keine Einschränkung der Gemeingültigkeit bedeutet, wenn wir voraussetzen, dass $l$ kleiner als $q$ ist, und dass $l$ und $q$ teilerfremde Zahlen sind. Laut dessen, was in M. K. I $\S 2$ angeführt worden ist, können dabei nur die folgenden Zahlen als erste Gelenkzahlen auftreten, nämlich

$$
-\left[-\frac{q k+l-q}{2 q}\right],-\left[-\frac{q k+l-q}{2 q}\right]+1, \quad \cdots k-1,
$$

denn $-\left[-\frac{q k+l-q}{q}\right]-1$ wird diesfalls gleich $k-1$. Der erste Gelenknenner $r_{1}$ wird durch folgende Gleichung bestimmt

$$
\frac{q k+l-q}{q}=k-\varkappa+\frac{k-\varkappa}{r_{1}},
$$

wo die untere Grenze der ganzen Zahl $\varkappa$ gleich 1 ist. Es ergibt sich

$$
r_{1}=\frac{(k-x) q}{q k+l-q-(k-x) q}=\frac{(k-x) q}{q(\varkappa-1)+l} .
$$

Für $\varkappa \geqq 2$ ergibt sich hieraus $r_{1}<k-\varkappa$; jede fast-monotone Entwicklung der Zahl $r_{1}$ kann wie oben ohne Monotonieabbruch verwertet werden und fastmonotoner Abschluss bei dem ersten Gelenk ist ausgeschlossen. Nur im Fall $\varkappa=1$, d. h. $r_{1}=\frac{(k-1) q}{l}$, ist eine besondere Untersuchung nötig. 


\section{F. RYDE, Fast-monotone Kettenbrüche}

Wenn dann $\frac{l}{q}<\frac{1}{2}$ ist, so ergibt sich $r_{1}>2(k-1)$. Wenn $r_{1}$ ganz ist, liegt dann fast-monotoner Abschluss vor. Sonst folgt Monotonieabbruch. Denn die erste Gelenkzahl bei jeder fast-monotonen Entwicklung einer Zahl $r_{1}$ muss grösser als $k-1$ sein, wenn $r_{1}$ grösser als $2(k-1)$ ist. $r_{1}=\frac{(k-1) q}{l}$ ist aber $\operatorname{gan} z$, wenn und nur wenn $k-1 \equiv 0(\bmod l)$ ist, da $l$ und $q$ teilerfremde Zahlen sind. Das oben Angeführte erklärt das Auftreten des Gliedes $\left\{\frac{k-1}{l}\right\}$ in der Formel $\left(A_{3}\right)$. - - $\mathrm{Zu}$ demselben Ergebnis führt auch eine Auseinandersetzung, die den letzten Satz in I zum Ausgangspunkt hat. - Die Formel $\left(A_{3}\right)$ behält ihre Gültigkeit auch in den Fällen $k=2$ und 3. Doch fällt jetzt die Summe auf der rechten Seite fort.

Wenn dagegen $\frac{k-1}{k} \leqq \frac{l}{q}<1$ und $k \geqq 2$ angenommen wird, so ergibt sich $r_{1}=\frac{(k-1) q}{l} \leqq k$; jede fast-monotone Entwicklung der Zahl $r_{1}$ kann jetzt ohne Monotonieabbruch verwertet werden, denn die erste Gelenkzahl jeder solcher Entwicklung muss kleiner als $k$ sein. Fast-monotoner Abschluss ist ausgeschlossen, denn $r_{1}$ wird nicht grösser als $2(k-1)$. Daher gilt jetzt die Formel (B).

Wenn schliesslich $\frac{1}{2} \leqq \frac{l}{q}<\frac{k-1}{k}$ und daher $k \geqq 3$ angenommen wird, so ergibt sich $k<\frac{(k-1)}{l} \underline{q} \leqq 2(k-1)$. Laut dessen, was in M. K. I $\S 2$ angeführt worden ist, können dabei nur die folgenden Zahlen als zweite Gelenkzahlen auftreten, nämlich

$$
-\left[-\frac{(k-1) q}{2 l}\right],-\left[-\frac{(k-1) q}{2 l}\right]+1, \quad \cdots-\left[-\frac{(k-1) q}{l}\right]-1 .
$$

Die letzte Zahl ist gewiss $\geqq k$, denn aus $k<\frac{(k-1) q}{l}$ folgt $-\frac{(k-1) q}{l}<-k$ und daher $\left[-\frac{(k-1) q}{l}\right] \leqq-k-1$ und mithin $-\left[-\frac{(k-1) q}{l}\right] \geqq k+1$. Diese letzte Zahl ist mithin ausgeschlossen, denr die zweite Gelenkzahl darf nicht grösser als $k-1$ sein. Da die letzte $Z$ ahl in vorstehender Reihe die einzige ist, die zu fast-monotonem Abschluss führen kann (vgl. die Auseinandersetzung bei der ersten Gelenkzahl, die gemeingültig ist), und da Monotonieabbruch bei dem zweiten Gelenk für $r_{1}$ ebenso nur bei dieser letzten Zahl vorkommen kann, so ergibt sich, da weiterhin

$$
\frac{(k-1) q}{l}=t+\frac{t}{(k-1) q-t l}
$$

ist, die Formel (C). 


\section{IV}

\section{Tafel der fast-monotonen Kettenbrüche.}

Die nachstehende Tafel der fast-monotonen Kettenbrüche ist mit Hilfe der Rekursionsformeln in III berechnet worden. Sie gibt die Anzahl $N m^{\prime}\left(\frac{p-q}{q}\right)$ der Entwicklungen der rationalen Zahl $\stackrel{p-q}{-\cdots}$ in der Form fast-monotoner Kettenbrüche für positive, ganzwertige $p \leqq 50$ und positive, ganzwertige $q \leqq p-1$. Wo kein Wert angegeben ist, bedeutet dies, dass $N m^{\prime}\left(\frac{p-q}{q}\right)$ hier gleich Null ist. Die Tafel enthält auch die bezüglichen Entwicklungen selbst für jede Bruchzahl $\frac{p-q}{q}$, wo $p$ und $q$ wie oben eingeschränkt sind. ${ }^{1}$ Wenn $\frac{p-q}{q}$ eine ganze Zahl, $n$, bedeutet, wächst $N m^{\prime}(n)$ rasch mit $n$. Daher sind die Entwicklungen diesfalls nur für $n<20$ angegeben. Wo keine Entwicklung angegeben ist, obgleich $N m^{\prime}\left(\frac{p-q}{q}\right)$ nicht gleich Null ist, bedeutet dies, dass der Bruch $\frac{p-q}{q}$ abgekürzt werden kann. Die bezüglichen Entwicklungen können bei dem abgekürzten Bruch nachgeschlagen werden.

Die Tafel ist hinsichtlich der Anzahl der Entwicklungen mit Hilfe einer geometrischen Darstellung des bezüghichen Algorithmus gepriift worden. Diese Darstellung hat die Form eines Nomogramms, der grosse Ähnlichkeit mit dem entsprechenden Nomogramm in M. K. III zeigt.

$$
\begin{aligned}
& q \quad N m^{\prime}\left(\frac{2-q}{q}\right) \\
& 10 \\
& 10 \\
& N m^{\prime}\left(\frac{3-q}{q}\right) \\
& 20 \\
& N m^{\prime}\left(\frac{4-q}{q}\right) \\
& 10 \\
& N m^{\prime}\left(\frac{5-q}{q}\right) \\
& \begin{array}{ll}
\mathbf{1} & \mathbf{0} \\
\mathbf{2} & \mathbf{0} \\
\mathbf{3} & \mathbf{0}
\end{array} \\
& N m^{\prime}\left(\frac{6-q}{q}\right) \\
& 10 \\
& \mathbf{2} \quad \mathbf{0} \\
& \begin{array}{lll}
q & N m^{\prime}\left(\frac{7-q}{q}\right) \\
1 & 0 & \\
2 & 0 & \\
3 & 1 & \left(1_{1}: 3\right) \\
4 & 0 & \\
& & \\
& N m^{\prime} & \left(\frac{8-q}{q}\right) \\
& & \\
1 & 1 & \left(4_{1} 1_{1}: 3\right) \\
2 & 0 & \\
3 & 0 & \\
4 & 0 & \\
& & \\
& N m^{\prime}\left(\frac{9-q}{q}\right) \\
& & \\
1 & 1 & \left(7_{1} 1_{1} 1_{1}: 3\right) \\
2 & 1 & \left(2_{1} 1_{1}: 3\right) \\
3 & 0 & \\
4 & 1 & \left(1_{1}: 4\right) \\
5 & 0 &
\end{array} \\
& { }_{1}\left(3_{1} 1_{2}: 4\right) \text { bedeutet hierbei } 3+\frac{3 \mid}{\mid 1}+\frac{1 \mid}{\mid 1}+\frac{1 \mid}{\mid 4} \text { usw. }
\end{aligned}
$$




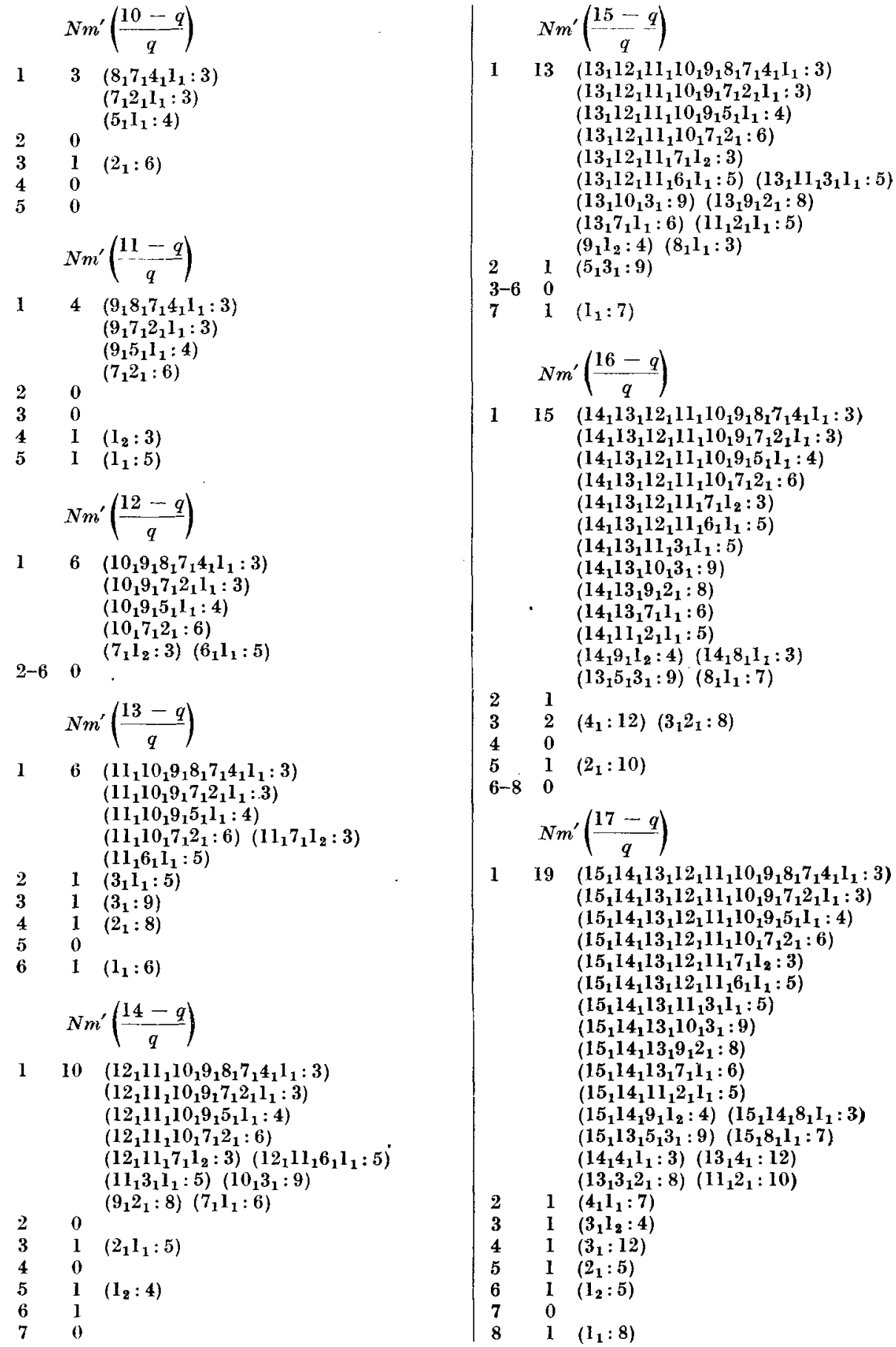




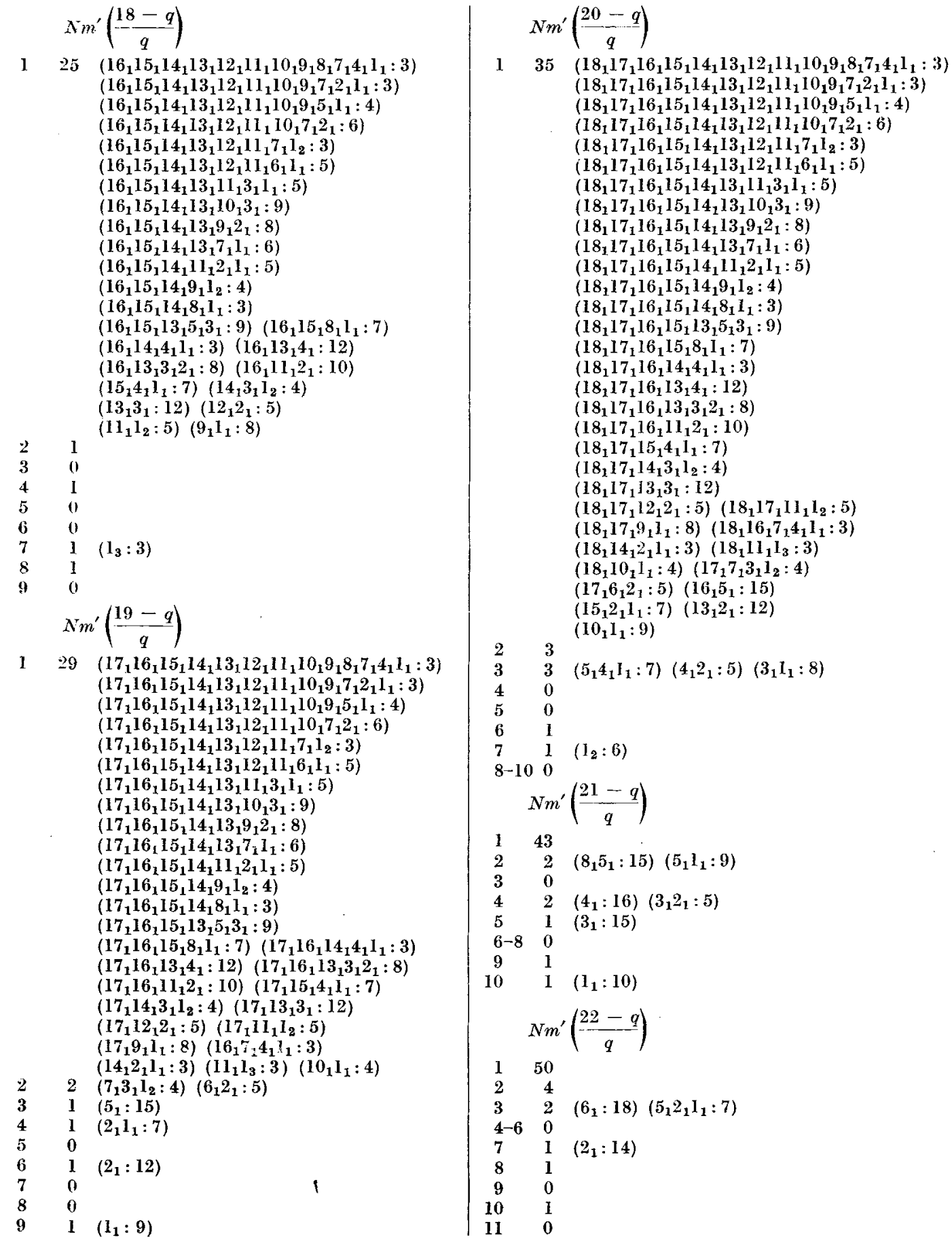


F. RYDE, Fast-monotone Kettenbrüche




ARKIV FöR MATEMATIK. Bd 1 nr 4
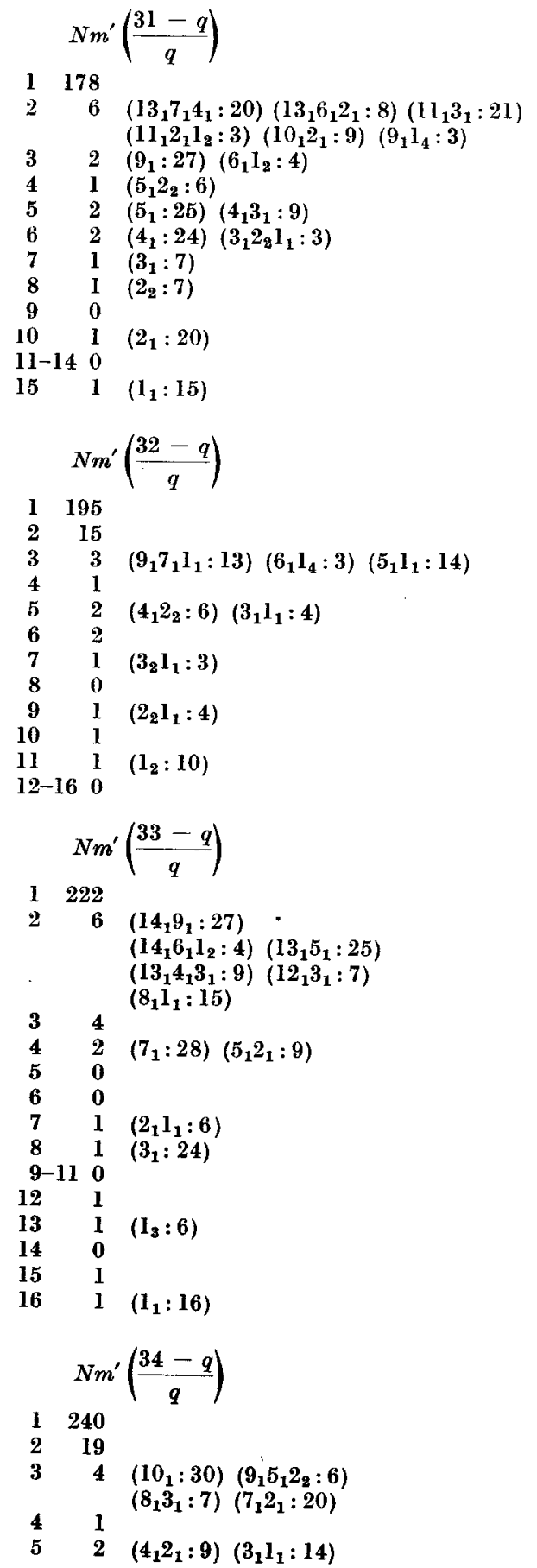
F. RYDE, Fast-monotone Kettenbriiche
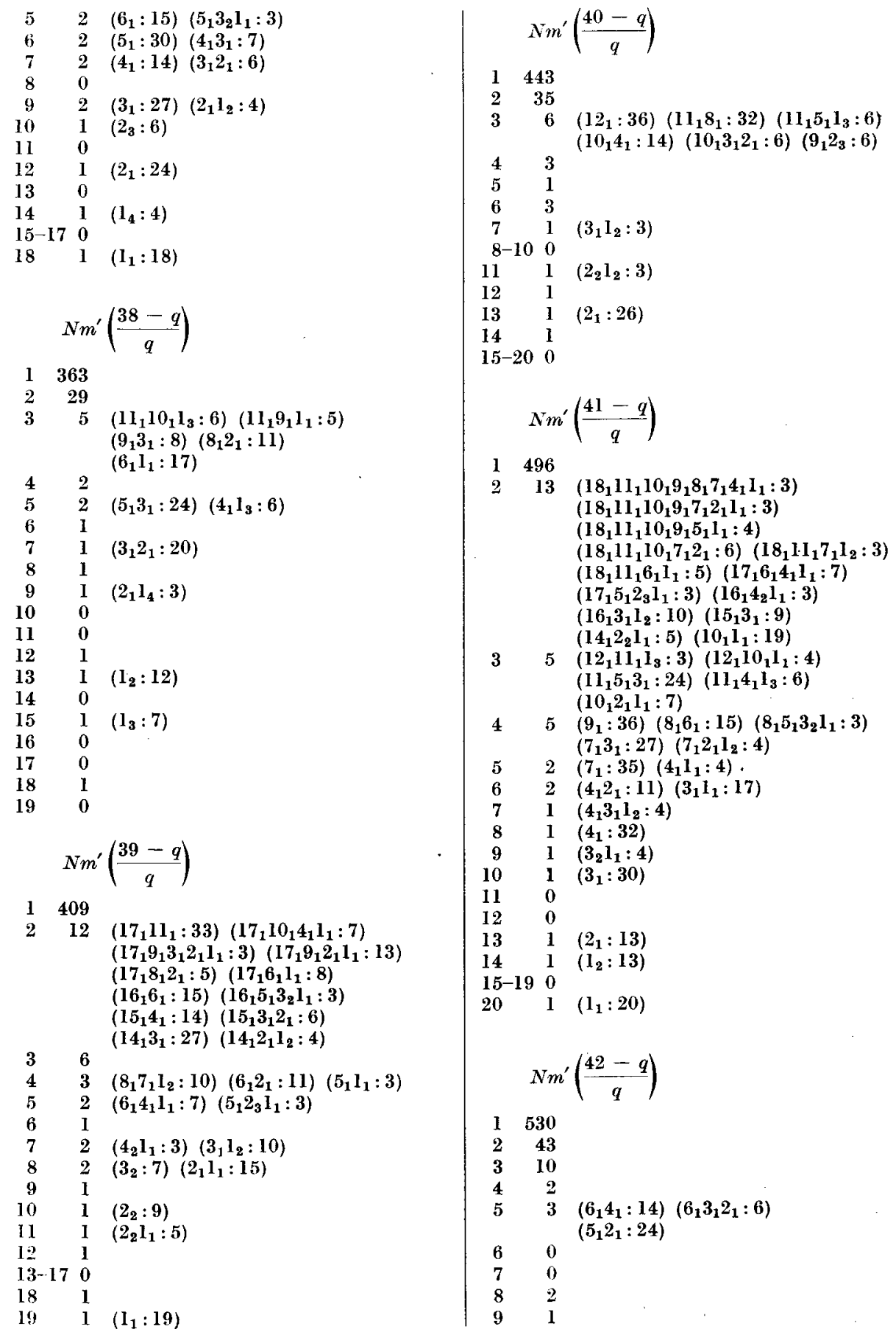


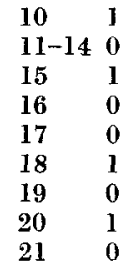

$$
N m^{\prime}\left(\frac{43-q}{q}\right)
$$$$
1595
$$$$
210\left(39_{1} 12_{1} 11_{1} l_{3}: 3\right)\left(19_{1} 12_{1} 10_{1} l_{1}: 4\right)
$$$$
\left(19_{1} 11_{1} 5_{1} 3_{1}: 24\right)\left(19_{1} 11_{1} 4_{1} 1_{3}: 6\right)
$$$$
\left(19_{1} 10_{1} 2_{1} 1_{1}: 7\right)\left(18_{1} 7_{1}: 35\right)
$$$$
\left(18_{1} 4_{1} 1_{1}: 4\right)\left(17_{1} 4_{1} 3_{1} 1_{2}: 4\right)
$$$$
\left(16_{1} 3_{2} 1_{1}: 4\right)\left(14_{1} 2_{1}: 13\right)
$$

$36 \quad\left(13_{1}: 39\right)\left(12_{1} 8_{1} 7_{1} 4_{1} l_{1}: 3\right)$

$$
\left(127_{1} 2_{1} 1_{1}: 3\right)\left(125_{1} 1_{1}: 4\right)
$$$$
\left(11_{1} 3_{1} 1_{2}: 3\right)\left(9_{1} 2_{1}: 26\right)
$$

$44 \quad\left(8_{1} 4_{2} 1_{1}: 3\right)\left(8_{1} 3_{1} 1_{2}: 10\right)$

$$
\left(7_{1} 2_{2} 1_{1}: 5\right)\left(5_{1} 1_{1}: 19\right)
$$

$54 \quad\left(76_{1} 1_{1}: 17\right)\left(62_{1} 1_{1}: 7\right)$

$$
\left(5_{1} l_{2}: 12\right)\left(4_{1} l_{1}: 9\right)
$$

$63\left(6_{1}: 36\right)\left(5_{1} 4_{1}: 14\right)\left(5_{1} 3_{1} 2_{1}: 6\right)$

$72 \quad\left(5_{1}: 35\right)\left(4_{1} 2_{1} l_{1}: 3\right)$

$81 \quad\left(32_{1}: 11\right)$

$93 \quad\left(3_{2} 2_{1} l_{1}: 3\right)\left(32_{1} l_{1}: I 3\right)\left(21_{1}: 8\right)$

$102\left(3_{1}: 10\right)\left(2_{1} 1_{3}: 6\right)$

$\begin{array}{lll}11 & 1 & \left(2_{2}: 10\right)\end{array}$

12

13

$\begin{array}{lll}14 & 1 & (2,: 28)\end{array}$

150

160

$\begin{array}{lll}17 & 1 & \left(1_{3}: 8\right)\end{array}$

$18-200$

$21 \quad 1 \quad\left(1_{1}: 21\right)$

$$
\begin{array}{crl} 
& N m^{\prime}\left(\frac{44-q}{q}\right) \\
& & \\
1 & 634 & \\
2 & 50 & \\
3 & 6 & \left(13_{1} 10_{1} 1_{1}: 19\right)\left(12_{1} 7_{1}: 35\right) \\
& & \left(12_{1} 4_{1} 1_{1}: 4\right)\left(11_{1} 4_{1}: 32\right) \\
& & \left(9_{1} 1_{2}: 13\right)\left(7_{1} 1_{1}: 20\right) \\
4 & 4 & \\
5 & 4 & \left(7_{1} 6_{1} 2_{1}: 11\right)\left(7_{1} 5_{1} 1_{1}: 3\right) \\
& & \left(6_{1} 3_{1}: 9\right)\left(4_{1} 1_{1}: 19\right) \\
6 & 2 & \\
7 & 2 & \left(4_{1} 3_{1}: 27\right)\left(4_{1} 2_{1} 1_{2}: 4\right) \\
8 & 0 & \\
9 & 2 & \left(3_{2}: 8\right)\left(2_{1} 1_{1}: 17\right) \\
10-13 & 0 & \\
14 & 1 & \\
15 & 1 & \left(1_{2}: 14\right) \\
16 & 1 & \\
17-19 & 0 &
\end{array}
$$

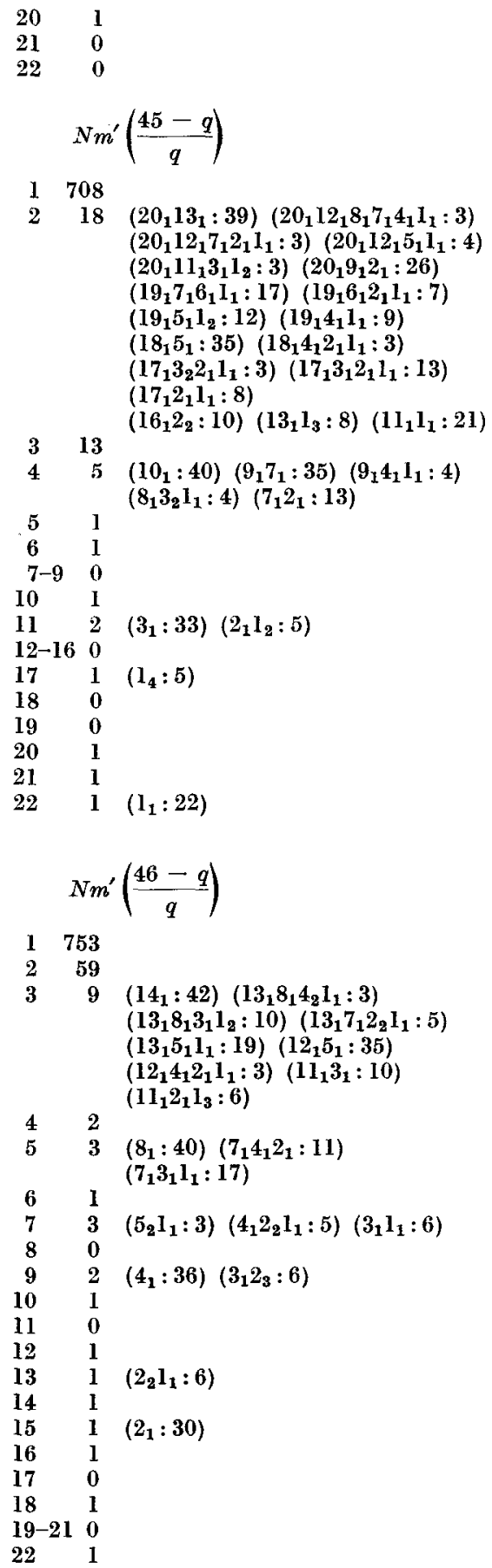




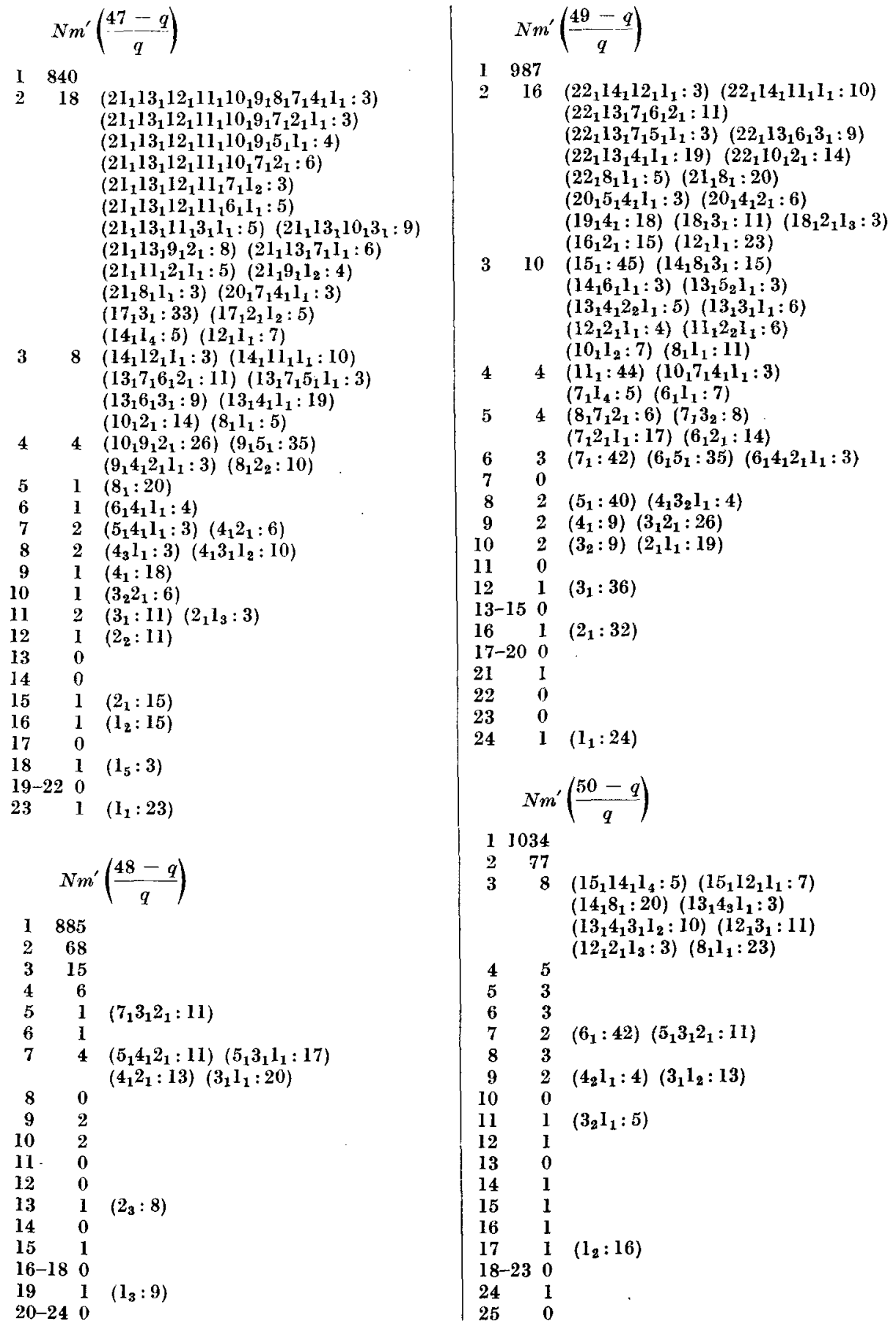

